\title{
Effect of manual lymph drainage in addition to guidelines and exercise therapy on arm lymphoedema related to breast cancer: randomised controlled trial
}

\author{
(a) (1) $\Theta$ OPEN ACCESS
}

\author{
Nele Devoogdt doctor in rehabilitation science ${ }^{12}$, Marie-Rose Christiaens professor, breast surgeon, \\ and coordinator ${ }^{3}$, Inge Geraerts research fellow ${ }^{1}$, Steven Truijen scientific coordinator ${ }^{2}$, Ann Smeets \\ breast surgeon ${ }^{3}$, Karin Leunen gynaecological oncologist ${ }^{3}$, Patrick Neven professor in gynaecological \\ oncology ${ }^{3}$, Marijke Van Kampen professor in rehabilitation science ${ }^{1}$
}

${ }^{1}$ Department of Rehabilitation Sciences, Katholieke Universiteit Leuven and Department of Physiotherapy, University Hospitals Leuven, Leuven, Belgium; ${ }^{2}$ Department of Health Care, Artesis University College of Antwerp, Antwerp; ${ }^{3}$ Multidisciplinary Breast Centre, University Hospitals Leuven and Faculty of Medicine, Katholieke Universiteit Leuven, Leuven

\begin{abstract}
Objective To determine the preventive effect of manual lymph drainage on the development of lymphoedema related to breast cancer.

Design Randomised single blinded controlled trial.

Setting University Hospitals Leuven, Leuven, Belgium.

Participants 160 consecutive patients with breast cancer and unilateral axillary lymph node dissection. The randomisation was stratified for body mass index (BMI) and axillary irradiation and treatment allocation was concealed. Randomisation was done independently from recruitment and treatment. Baseline characteristics were comparable between the groups.

Intervention For six months the intervention group $(\mathrm{n}=79)$ performed a treatment programme consisting of guidelines about the prevention of lymphoedema, exercise therapy, and manual lymph drainage. The control group $(n=81)$ performed the same programme without manual lymph drainage.

Main outcome measures Cumulative incidence of arm lymphoedema and time to develop arm lymphoedema, defined as an increase in arm volume of $200 \mathrm{~mL}$ or more in the value before surgery.

Results Four patients in the intervention group and two in the contro group were lost to follow-up. At 12 months after surgery, the cumulative incidence rate for arm lymphoedema was comparable between the intervention group (24\%) and control group (19\%) (odds ratio 1.3, 95\% confidence interval 0.6 to $2.9 ; \mathrm{P}=0.45$ ). The time to develop arm lymphoedema was comparable between the two group during the first year after surgery (hazard ratio $1.3,0.6$ to $2.5 ; \mathrm{P}=0.49$ ). The sample size calculation was based on a presumed odds ratio of 0.3 , which is not included in the $95 \%$ confidence interval. This odds ratio was calculated as (presumed cumulative incidence of lymphoedema in intervention
\end{abstract}

group/presumed cumulative incidence of no lymphoedema in intervention group) $\times$ (presumed cumulative incidence of no lymphoedema in control group/presumed cumulative incidence of lymphoedema in control group) or $(10 / 90) \times(70 / 30)$.

Conclusion Manual lymph drainage in addition to guidelines and exercise therapy after axillary lymph node dissection for breast cancer is unlikely to have a medium to large effect in reducing the incidence of arm lymphoedema in the short term.

Trial registration Netherlands Trial Register No NTR 1055.

\section{Introduction}

Worldwide, breast cancer is the most common cancer in women. Detection and treatment of breast cancer have significantly improved over past decades, which results in higher survival rates. ${ }^{1}$ More attention is now therefore paid to complications related to treatment, such as arm lymphoedema.

For a woman with breast cancer, lymphoedema is a debilitating and incurable problem that is caused by reduced transport capacity of the lymph system (related to the surgery or radiotherapy, or both), sometimes combined with an increase in lymph load (related to hypertension, for example). ${ }^{23}$ Twelve months after axillary lymph node dissection, the point prevalence of arm lymphoedema ranges from $12 \%{ }^{4}$ to $26 \%,{ }^{5}$ though some have reported point prevalence rates up to $70 \% .^{6}$ This wide variety is related to differences in treatment of breast cancer, methods of measurement, delay in measuring, and definition of lymphoedema. ${ }^{7}$ Use of the most accurate and reliable method for assessment is crucial to advance our understanding of preventive strategies. ${ }^{8}$ To register the natural difference between the dominant and non-dominant arm, volume 
and circumferences of both arms have to be assessed before axillary lymph node dissection. ${ }^{9}$ Commonly used definitions for arm lymphoedema, taking into account preoperative differences, are a change of $200 \mathrm{~mL}$ or more in volume ${ }^{10}$ or a change in circumference of $2 \mathrm{~cm}$ or more at two adjacent measurement sites, ${ }^{11}$ both compared with the preoperative value, together with a subjective feeling of swelling. ${ }^{9}$ Around $67-80 \%$ of patients with lymphoedema develop arm swelling within the first year after axillary lymph node dissection. ${ }^{12}{ }^{13}$ Possible risk factors are being overweight, radiotherapy (of the axilla or supraclavicular region, or both), and the number and levels of axillary lymph nodes dissected. ${ }^{14-16}$

Prevention of lymphoedema after axillary dissection for breast cancer is poorly investigated. Recently, one study showed that a combination of guidelines, exercise therapy, and manual lymph drainage was more effective in preventing arm lymphoedema than just guidelines. ${ }^{11}$ In another study, fewer patients who received guidelines and exercise therapy after axillary dissection developed lymphoedema than patients without treatment, although this difference was not significant. ${ }^{10}$ As far as we are aware, the preventive effect of manual lymph drainage on the development of lymphoedema has not been investigated in a randomised controlled trial. ${ }^{17}$ Manual lymph drainage is an integral part of lymphoedema treatment. It is a gentle massage technique and has different purposes: stimulating resorption of lymph by the lymph capillaries, increasing lymph transport from the hand to axilla by existing lymph collaterals, and creating lymph collateral pathways between the arm and trunk and between both axillas (that is, the Mascagni pathway and axilloaxillary anastomoses). ${ }^{18} 19$

We compared the effect of a treatment programme consisting of guidelines, exercise therapy, and manual lymph drainage (intervention group) and the same programme without lymph drainage (control group) on the development of lymphoedema related to breast cancer.

\section{Methods \\ Participants}

Between October 2007 and February 2009, all patients with operable breast cancer and scheduled for unilateral surgery at the multidisciplinary breast centre of the University Hospitals in Leuven were assessed before surgery. After surgery, patients with an axillary lymph node dissection were asked to participate in the study $(\mathrm{n}=337)$, and $160(48 \%)$ agreed to participate. All included patients gave written informed consent.

\section{Procedure}

Patients were randomised into the intervention group (receiving guidelines about the prevention of lymphoedema, exercise therapy, and manual lymph drainage) or a control group (receiving guidelines and exercise therapy). Patients received guidelines about the prevention of lymphoedema and exercise therapy as soon as possible after surgery. Manual lymph drainage (in the intervention group) or no lymph drainage (control group) was started one week after removal of the axillary drains, so five weeks after the surgery on average, and was applied over 20 weeks. In our hospital, there is a standard procedure to leave the drains in until less than $30 \mathrm{~mL} /$ day is collected, and on average this takes four weeks. Randomisation occurred after we had information about the adjuvant treatment decided on at the multidisciplinary oncological consultation (three weeks after surgery). Randomisation was performed within each stratum by using permuted blocks (size $=4)$. Strata were body mass index (BMI; $\leq 25 v>25$ ) and postoperative axillary irradiation (yes/no) because these factors are the two most important risk factors for development of arm lymphoedema after axillary dissection. ${ }^{14}{ }^{16}$ The allocation to the treatment groups was concealed. A different person from the one doing the recruitment and treatments of patients carried out the randomisation. The sequence of randomisation was determined by the patient's identification number, which she/he received after inclusion in the study.

\section{Interventions}

All patients received guidelines about the prevention of arm lymphoedema ${ }^{20}$ : lift the arm in case of heaviness, avoid lifting heavy objects and performing repetitive movements, use the arm as normally as possible, avoid limb constriction, avoid extremes of temperature, apply skin care, wear a sleeve during a flight, and avoid an increase in weight. Patients received a brochure with these guidelines and, on request, received extra information during the exercise therapy sessions.

The exercise therapy consisted of different treatment modalities. Table $1 \Downarrow$ gives an overview of the different modalities, their purpose, and method. Each session was individual and took half an hour. At the start of the treatment, patients had to come twice a week. Later, when the difference in shoulder mobility compared with the value before surgery was less than $20^{\circ}$, frequency was reduced to once a week, and then, if the patient was able to start maintenance treatment, to once every two weeks.

Patients in the intervention group also received standardised manual lymph drainage. Firstly, lymph nodes of neck and axilla were emptied. Secondly, axilloaxillary anastomoses at the breast and back and lymphatics at the lateral side of the shoulder (Mascagni pathway) were stimulated. Thirdly, the arm and hand were drained from proximal to distal. One session took half an hour. Patients were scheduled to receive 40 sessions of manual lymph drainage, with an increase in frequency from once a week to three times a week, and then a decrease to once a week, to create a gradual adaptation of the lymph system and not to end too abruptly.

Four therapists performed all treatments, including providing guidelines, exercise therapy, and manual lymph drainage. Two therapists had more than 10 years' experience in treatment of lymphoedema and lymph drainage. One had followed courses at the Vodder School Belgium, and the other was educated in lymph drainage at the University of Brussels. They performed more than $70 \%$ of the lymph drainage sessions. The other two therapists were less experienced but underwent training in our centre before the start of the study. At several times during the study, training sessions were organised to ensure standardisation and similarity of the manual lymph drainage.

If a patient in either group developed arm lymphoedema, defined as an increase of the arm volume of $200 \mathrm{~mL}$ or more, she or he had to wear an inelastic bandage until the lymphoedema was maximally diminished and thereafter had to wear a custom-made sleeve.

\section{Assessments}

Information about the breast surgery, axillary surgery, and adjuvant treatment was collected from the medical file of the patient.

All patients were measured before and 1, 3, 6, and 12 months after axillary surgery in the department of physiotherapy. Patients with a subjective feeling of arm lymphoedema received an extra measurement of the arm. Two blinded and well trained 
assessors (different from the therapists) performed the measurements. Assessors were especially trained for the study during one month. Patients were explicitly asked not to mention their group allocation to the assessor.

Table 2 shows an overview of the primary and secondary outcome parameters and the assessment method $\Downarrow$.

At each measurement session (before and after surgery), we assessed the volume and circumference of both arms and body weight. Volume of the arm was measured by the water displacement method with a volumeter up to the reference point $16 \mathrm{~cm}$ proximal to the olecranon. ${ }^{21} \mathrm{We}$ developed a reliable and valid measurement device, consisting of a stainless steel bar on which a tapeline was fixed every $4 \mathrm{~cm}$, to measure arm circumference at the olecranon and 4, 8, 12, 16, and $20 \mathrm{~cm}$ above and under the olecranon. Inter-rater reliability of the arm measurements performed by our assessors ranged between 0.94 and 0.99 , depending on the measurement side. ${ }^{22}$ We used two different definitions for objective lymphoedema: an increase of $200 \mathrm{~mL}$ or more in the difference in the arm volume between the affected and healthy side compared with the difference before surgery and an increase of $2 \mathrm{~cm}$ or more in the difference in arm circumference between the affected and healthy side at two or more adjacent measurement points compared with the difference before surgery. Body weight was measured with an electronic balance.

Furthermore, at each measurement session after surgery the patient filled in a questionnaire we had developed to score the presence of subjective arm lymphoedema. At 3, 6, and 12 months after surgery, the patient filled in the SF-36 to measure health related quality of life..$^{23}$

\section{Sample size and statistical analyses}

We performed power calculations before the start of the study. If $10 \%$ of the patients in the intervention group and $30 \%$ of the patients in the control group developed arm lymphoedema during the first year after the surgery and we applied a power of $80 \%$ and $\alpha$ of 0.05 , we would need a minimum of 146 patients. Taking into account the dropouts $(10 \%)$, we had to include 160 patients. Two studies have reported a cumulative incidence rate of lymphoedema of $26 \%$ (160/631 patients with breast cancer $)^{13}$ and $40 \%$ (125/287 patients with breast cancer $)^{8}$ 12 and 18 months after the surgery, which is comparable with our estimated rate for the control group. As no previous studies have examined the additional effect of manual lymph drainage on the prevention of arm lymphoedema, we discussed the effect with experienced physical therapists, and they considered that only $10 \%$ of the patients receiving guidelines, exercise therapy, and manual lymph drainage would develop arm lymphoedema during the first year after surgery.

We compared patients' characteristics between the excluded and included patients to analyse the representativeness of our study group. We used the independent $t$ test for continuous data, Mann-Whitney $U$ test for ordinal data, and $\chi^{2}$ test for nominal data. Data were analysed according the intention to treat principle. We used logistic regression analyses to compare the cumulative incidence and point prevalence of objective lymphoedema and point prevalence of subjective lymphoedema at 3, 6, and 12 months between the intervention and control group. ${ }^{24}$ Cumulative incidence is the rate of occurrence during a specific time period, whereas point prevalence indicates the occurrence of a disease at a particular point in time.

We used Kaplan-Meier survival analyses (with log rank test) to compare groups concerning time to event (for both definitions of arm lymphoedema). ${ }^{25}$ At 3,6 , and 12 months, we compared the change in the absolute difference in arm volume with the value before surgery and quality of life between the two groups with Mann-Whitney U test because the variables were not normally distributed. All data were analysed with SPSS 17.0.

\section{Results}

Compared with non-participating (excluded) patients, included patients were 2.8 years younger $(\mathrm{P}=0.04)$, had 1.3 higher $\mathrm{BMI}$ $(\mathrm{P}=0.02)$, less often received axillary irradiation $(8 \% v 16 \%$; $\mathrm{P}=0.03)$, and more often received chemotherapy (68\% v 58\%; $\mathrm{P}=0.06)$. All other characteristics related to disease and treatment, such as number and levels of lymph nodes dissected, type of breast surgery, surgery at the dominant side, tumour size, lymph node stage, radiotherapy of the internal mammary chain and medial supraclavicular region, trastuzumab and endocrine treatment, were comparable between groups (data not shown).

From 160 included patients, 79 were randomised to the intervention group and 81 to the control group. Some 158 patients $(99 \%)$ were measured at one, three, and six months after surgery (77 in the intervention group and 81 in the control group) and 154 patients (96\%) at 12 months after surgery (75 and 79). Figure 1 shows the flow of patients and reasons for dropping out $\Downarrow$.

All characteristics of the two groups were comparable (table $3 \Downarrow$ ). Four patients in the intervention group and two in the control group had developed arm lymphoedema (increase of $200 \mathrm{~mL}$ or more) before the start of the 20 week treatment period. Two patients in the intervention group and three in the control group developed deep venous thrombosis in the healthy $\mathrm{arm}$. The two patients with thrombosis in the intervention group had $-2.4 \mathrm{~kg}$ and $2.9 \mathrm{~kg}$ change of weight during follow-up. The three patients with thrombosis in the control group gained $3.2-6.8 \mathrm{~kg}$ in weight.

Eleven patients of the intervention group (15\%) received 23-29 manual lymph drainage sessions, 26 patients $(36 \%)$ received 30-35 sessions, and 36 patients (49\%) received more than 35 sessions. The main reason for absence during the therapy sessions was illness related to chemotherapy and radiotherapy. Other reasons were problems with transport, holiday, and illness from other cause.

\section{Primary outcomes}

Compared with controls, patients in the intervention group had a comparable cumulative incidence rate for arm lymphoedema defined as an increase of $200 \mathrm{~mL}$ or more 12 months after surgery (18 (24\%) in intervention group $v 15(19 \%)$ in control group; odds ratio $1.3,95 \%$ confidence interval 0.6 to 2.9 ; $\mathrm{P}=0.45$ ). Both groups also had a comparable cumulative incidence for arm lymphoedema at three and six months after surgery (table $4 \Downarrow$ ). The adjusted odds ratio (corrected for risk factors for lymphoedema such as BMI, radiotherapy of axilla and radiotherapy of internal mammary chain and medial supraclavicular region, and number and levels of axillary lymph nodes dissected) was comparable with the unadjusted odds ratio (data not shown).

Figure 2 shows the Kaplan-Meier survival analyses for time to develop arm lymphoedema $\downarrow$. Time to develop arm lymphoedema defined as an increase of $200 \mathrm{~mL}$ or more was comparable between the two groups during the first year after surgery (hazard ratio $1.3,0.6$ to $2.5 ; \log$ rank $\mathrm{P}=0.44$ ). The survival analyses corrected for the lymphoedema risk factors gave comparable results. 


\section{Secondary outcomes}

At 3, 6, and 12 months after surgery, the cumulative incidence rate for arm lymphoedema and time to develop arm lymphoedema (defined as increase of $\geq 2 \mathrm{~cm}$ at two or more adjacent measurement points), the point prevalence of arm lymphoedema (defined as increase of $\geq 200 \mathrm{~mL}$ or increase of $\geq 2 \mathrm{~cm}$ ), and the point prevalence of subjective lymphoedema were comparable between the two groups (table $4 \Downarrow$ ).

At each follow-up both groups had a similar increase of arm volume compared with the level before surgery. They also had a comparable mental and physical health related quality of life (table $5 \Downarrow$ ).

Figures $3 \Downarrow$ and $4 \Downarrow$ show the distribution of the changes in arm volume and the maximal increase in arm circumference at two adjacent measurement points 12 months after surgery. In both groups, the distributions are comparable. The same applied to the results for mental and physical health related quality of life (data not shown).

\section{Discussion}

A six month treatment programme consisting of guidelines, exercise therapy, and manual lymph drainage applied after axillary lymph node dissection for breast cancer had no medium to large effect on the prevention of arm lymphoedema than the same treatment programme without manual lymph drainage. The cumulative incidence of arm lymphoedema and time to develop arm lymphoedema-defined as an increase of $200 \mathrm{~mL}$ or more-were comparable between both groups (primary outcomes), during, immediately after, and six months after the treatment sessions. The secondary outcomes were also comparable between both groups.

\section{Comparison with other studies}

We could not find a previous randomised controlled trial on the preventive effect of manual lymph drainage on the development of lymphoedema related to breast cancer. We found only a congress report, but the methods of the study were minimally described. ${ }^{26}$ They concluded that manual lymph drainage was effective for the prevention of arm lymphoedema. At five years after surgery, the point prevalence of arm lymphoedema, defined as an increase of $2 \mathrm{~cm}$ or more, was considerably higher: $30 \%$ in the group receiving manual lymph drainage and $45 \%$ in the control group, whereas in our study at 12 months after surgery the point prevalence was $15 \%$ and $9 \%$, respectively.

Lacomba et al showed a preventive effect of a combination of manual lymph drainage and exercise therapy on the development of lymphoedema. ${ }^{11}$ They included 120 patients after axillary dissection, although power calculation was performed retrospectively on the basis of an estimated cumulative incidence of $10 \%$ in the intervention group and $30 \%$ in the control group and a power of $70 \%$. Patients were treated over three weeks. Within the first year after surgery, $7 \%$ of the patients in the intervention group, who received guidelines, exercise therapy, and manual lymph drainage, and $25 \%$ of the patients in the control group, who received only guidelines, had developed arm lymphoedema. All patients who developed arm lymphoedema did so 6-12 months after surgery. In our study we performed a comparable power calculation, but we did this before the start of the study, and our power was higher (80\%). In patients in our intervention group, who also received guidelines, exercise therapy, and manual lymph drainage, the cumulative incidence rate was much higher (24\%). In our control group, who received guidelines and exercise therapy, the cumulative incidence rate was comparable (19\%). In our study, the patients developed their arm swelling earlier. In contrast with Lacomba et al, only $36 \%$ of the patients with arm lymphoedema (13/36) developed oedema 6-12 months after surgery. We cannot explain this discrepancy. Our patients were treated for a longer time (six months versus three weeks). Perhaps our study population was at higher risk of developing lymphoedema because of more extensive axillary surgery or because of more frequent irradiation of the axilla. This information is missing in the study of Lacomba et al. ${ }^{11}$

\section{Strengths and limitations}

The present study had several strengths. The volume and circumference of both arms were measured before surgery to determine the natural difference in arm volume. ${ }^{27}$ At each follow-up, we compared the evolution of the difference in arm volume between the affected and the healthy arm to correct for patients' weight gain. In some cases, the healthy arm can be swollen because of a deep venous thrombosis after implantation of a central venous catheter for chemotherapy. ${ }^{28}$ The proportion of patients undergoing chemotherapy before or after surgery, however, was comparable in our two groups. We carried out randomisation and stratification for BMI and axillary radiotherapy to reduce potential bias concerning the most important risk factors for lymphoedema at baseline. In addition, randomisation was concealed. Only six patients (4\%) dropped out after the start of the 20 week treatment period. Our study population received an average of 34 sessions of manual lymph drainage over 20 weeks, so we are confident that we examined the preventive effect of manual lymph drainage.

Our study also had several limitations. Six patients developed lymphoedema soon after axillary surgery and before the start of the 20 week treatment period. Our patients were not entirely representative of all patients with breast cancer, although compared with non-participants, they were only about 3 years younger, had a BMI about 1 higher, and fewer received axillary radiotherapy; all other characteristics were comparable. Half of the therapists were experienced in performing manual lymph drainage and half were less experienced. Their method of lymph drainage was comparable as they were especially trained before the study and standardisation and similarity of lymph drainage of the four therapists was controlled at several times during the study. In addition, the experienced therapists performed the major part of the lymph drainage sessions. Our patients did not receive the planned 40 manual lymph drainage sessions because of illness related to chemotherapy, although up to $85 \%$ of the patients received 30 sessions or more.

\section{Future research}

As we found no medium to large effect of manual lymph drainage (in addition to guidelines and exercise therapy) on the development of lymphoedema related to breast cancer, a future study should be performed to determine whether it has a small effect. The power calculation could be based on the combination of the cumulative incidence rates for arm lymphoedema from the study of Lacomba et al and our study, which leads to an estimated cumulative incidence of $16 \%((4+18) /(59+77))$ in the intervention group and $21 \%((14+15) /(57+81))$ in the control group. We need to continue to follow up our patients as $20-33 \%$ of patients with breast cancer who develop arm lymphoedema will do so more than 12 months after surgery.

Further research should confirm our results. Lymphoscintigraphy can be used to compare the lymph function on the affected side ${ }^{29}$ between the groups. Instead of circumference and volumetric 
measurements, lymphoedema can also be assessed with ultrasonography. This can directly measure thickening of cutis and subcutis because of lymphoedema and exclude changes in arm volume caused by muscular atrophy and hypertrophy. ${ }^{30} 31$

\section{Conclusion and policy implication}

Our results show that manual lymph drainage applied after axillary lymph node dissection for breast cancer and additional to guidelines and exercise therapy is unlikely to have a medium to large effect on the prevention of arm lymphoedema in the short term. It is important to inform patients of this after axillary dissection.

We thank Karen Vermeiren, Liesbeth Lamsens, Ann Schoonjans, and Sofie Elen for treating the patients and Hilde Lemkens and Ine Van Nuland for measuring the patients. We are grateful to the nurses of the department of surgical oncology and medical staff of the multidisciplinary breast clinic to motivate the patients to participate in our study. Finally, we thank all patients who took part in this study.

Contributors: ND, MVK, and M-RC conceived and designed the study. $\mathrm{M}-\mathrm{RC}, \mathrm{AS}, \mathrm{KL}$, and PN provided patients. Hilde Lemkens and Ine Van Nuland recruited the patients and performed all assessments. Karen Vermeiren, Liesbeth Lamsens, Ann Schoonjans, and Sofie Elen performed all treatments. ND, ST, and MVK analysed and interpreted the data. ND wrote the manuscript and is guarantor. All authors read and approved the final manuscript.

Funding: This study was funded by the agency for Innovation by Science and Technology (Applied Biomedical Research) (IWT 60519). The funding source had no role in study design, data collection, data analysis, data interpretation, or writing of the report.

Competing interests: All authors have completed the ICMJE uniform disclosure form at www.icmje.org/coi_disclosure.pdf (available on request from the corresponding author) and declare: no support from any organisation for the submitted work; no financial relationships with any organisations that might have an interest in the submitted work in the previous three years; no other relationships or activities that could appear to have influenced the submitted work.

Ethical approval: This study was approved by the ethics committee of the University Hospitals of Leuven responsible for human and animal experimentation (B-number: 32220072574, S-number: 50682), and all participants gave written informed consent.

Data sharing: No additional data available.

1 Berry DA, Cronin KA, Plevritis SK, Fryback DG, Clarke L, Zelen M, et al. Effect of screening and adjuvant therapy on mortality from breast cancer. N Engl J Med 2005;353:1784-92. Foldi E, Foldi M, Clodius L. The lymphedema chaos: a lancet. Ann Plast Surg 1989;22:505-15.

3 Stanton AW, Modi S, Mellor RH, Levick JR, Mortimer PS. Recent advances in breast cancer-related lymphedema of the arm: lymphatic pump failure and predisposing factors. Lymphat Res Biol 2009;7:29-45.

4 Fleissig A, Fallowfield LJ, Langridge $\mathrm{Cl}$, Johnson L, Newcombe RG, Dixon JM, et al. Post-operative arm morbidity and quality of life. Results of the ALMANAC randomised trial comparing sentinel node biopsy with standard axillary treatment in the management of patients with early breast cancer. Breast Cancer Res Treat 2006;95:279-93.
5 Karki A, Simonen R, Malkia E, Selfe J. Impairments, activity limitations and participation restrictions 6 and 12 months after breast cancer operation. J Rehabil Med 2005:37:180-8.

6 Armer JM, Stewart BR. A comparison of four diagnostic criteria for lymphedema in a post-breast cancer population. Lymphat Res Biol 2005;3:208-17.

7 Rietman JS, Dijkstra PU, Hoekstra HJ, Eisma WH, Szabo BG, Groothoff JW, et al. Late morbidity after treatment of breast cancer in relation to daily activities and quality of life: a systematic review. Eur J Surg Oncol 2003;29:229-38.

8 Hayes S, Janda M, Cornish B, Battistutta D, Newman B. Lymphedema secondary to breast cancer: how choice of measure influences diagnosis, prevalence, and identifiable risk factors. Lymphology 2008;41:18-28.

9 Armer JM. The problem of post-breast cancer lymphedema: impact and measurement issues. Cancer Invest 2005;23:76-83.

10 Box RC, Reul-Hirche HM, Bullock-Saxton JE, Furnival CM. Physiotherapy after breast cancer surgery: results of a randomised controlled study to minimise lymphoedema. Breast Cancer Res Treat 2002;75:51-64.

11 Lacomba MT, Sanchez MJY, Goni AZ, Merino DP, del Moral OM, Tellez EC, et al. Effectiveness of early physiotherapy to prevent lymphoedema after surgery for breast cancer: randomised, single blinded, clinical trial. BMJ 2010;340:b5396.

12 Clark B, Sitzia J, Harlow W. Incidence and risk of arm oedema following treatment for breast cancer: a three-year follow-up study. QJM 2005:98:343-8.

13 Norman SA, Localio AR, Potashnik SL, Simoes Torpey HA, Kallan MJ, Weber AL, et al. Lymphedema in breast cancer survivors: incidence, degree, time course, treatment, and symptoms. J Clin Oncol 2009;27:390-7.

14 Erickson VS, Pearson ML, Ganz PA, Adams J, Kahn KL. Arm edema in breast cancer patients. J Natl Cancer Inst 2001;93:96-111.

15 Hayes SB, Freedman GM, Li T, Anderson PR, Ross E. Does axillary boost increase lymphedema compared with supraclavicular radiation alone after breast conservation? Int J Radiat Oncol Biol Phys 2008;72:1449-55.

16 Paskett ED, Naughton MJ, McCoy TP, Case LD, Abbott JM. The epidemiology of arm and hand swelling in premenopausal breast cancer survivors. Cancer Epidemiol Biomarker Prev 2007;16:775-82.

17 Cheville A. Prevention of lymphoedema after axillary surgery for breast cancer. BMJ 2010;340:b5235.

18 Ferrandez JC, Laroche JP, Serin D, Felix-Faure C, Vinot JM. [Lymphoscintigraphic aspects of the effects of manual lymphatic drainage.] J Mal Vasc 1996;21:283-9.

19 Leduc A, Lievens P. [Experimental evidence for motoric rehabilitation in lymphoedema (author's transl).] Acta Chir Belg 1979;78:189-93.

20 Ridner SH. Breast cancer lymphedema: pathophysiology and risk reduction guidelines. Oncol Nurs Forum 2002;29:1285-93.

21 Gebruers N, Truijen S, Engelborghs S, De Deyn PP. Volumetric evaluation of upper extremities in 250 healthy persons. Clin Physiol Funct Imaging 2007;27:17-22.

22 Devoogdt N, Lemkens H, Geraerts I, Van Nuland I, Flour M, Coremans T, et al. A new device to measure upper limb circumferences: validity and reliability. Int Angiol 2010;29:401-7.

23 Ware JE Jr. SF-36 health survey update. Spine (Phila Pa 1976) 2000;25:3130-9

24 Chan YH. Biostatistics 202: logistic regression analysis. Singapore Med J 2004;45:149-53.

25 Chan YH. Biostatistics 203. Survival analysis. Singapore Med J 2004;45:249-56.

26 Partsch $\mathrm{H}$, ed. Post surgical physiotherapeutic treatment: interest in secondary upper limb lymphedemas prevention. XIth International Congress of Lymphology, 1988.

27 Stout Gergich NL, Pfalzer LA, McGarvey C, Springer B, Gerber LH, Soballe P. Preoperative assessment enables the early diagnosis and successful treatment of lymphedema. Cancer 2008:112:2809-19.

28 Kuter DJ. Thrombotic complications of central venous catheters in cancer patients. Oncologist 2004;9:207-16.

29 O'Mahony S, Solanki CK, Barber RW, Mortimer PS, Purushotham AD, Peters AM. Imaging of lymphatic vessels in breast cancer-related lymphedema: intradermal versus subcutaneous injection of 99mTc-immunoglobulin. Am J Roentgenol 2006:186:1349-55.

30 Mellor RH, Bush NL, Stanton AW, Bamber JC, Levick JR, Mortimer PS. Dual-frequency ultrasound examination of skin and subcutis thickness in breast cancer-related lymphedema. Breast $J$ 2004;10:496-503.

31 Van der Veen P, Vermeiren K, Von Kemp K, Lamote J, Sacre R, Lievens P. A key to understanding postoperative lymphoedema: a study on the evolution and consistency of oedema of the arm using ultrasound imaging. Breast 2001;10:225-30.

Accepted: 21 July 2011

\section{Cite this as: BMJ 2011;343:d5326}

This is an open-access article distributed under the terms of the Creative Commons Attribution Non-commercial License, which permits use, distribution, and reproduction in any medium, provided the original work is properly cited, the use is non commercial and is otherwise in compliance with the license. See: http://creativecommons.org/licenses/by$\mathrm{nc} / 2.0 /$ and http://creativecommons.org/licenses/by-nc/2.0/legalcode. 


\section{What is already known on this topic}

There is no standard definition to determine arm lymphoedema related to breast cancer

The combination of guidelines about the prevention of arm lymphoedema, exercise therapy, and manual lymph drainage is thought to be more effective than just guidelines

\section{What this study adds}

Manual lymph drainage in addition to guidelines about the prevention of lymphoedema and exercise therapy has no medium to large effect on the prevention of arm lymphoedema related to breast cancer in the short term

\section{Tables}

\begin{tabular}{|c|c|c|}
\hline Modality & Purpose & Method \\
\hline Mobilisation of shoulder & $\begin{array}{l}\text { To improve passive and active shoulder } \\
\text { mobility }\end{array}$ & $\begin{array}{l}\text { Average } 10 \text { minutes per session. Angular passive mobilisation of shoulder (especially anteflexion } \\
\text { and abduction) combined with traction/translation to prevent articular problems and impingement }\end{array}$ \\
\hline $\begin{array}{l}\text { Stretching breast } \\
\text { muscles }\end{array}$ & $\begin{array}{l}\text { To improve muscle flexibility and passive } \\
\text { and active shoulder mobility }\end{array}$ & $\begin{array}{l}\text { Average } 10 \text { minutes per session (together with scar tissue massage). Passive and active } \\
\text { stretching and transverse strain of major and minor pectoral muscle }\end{array}$ \\
\hline Scar tissue massage & To improve flexibility of scar tissue & $\begin{array}{l}\text { Mobilisation of scar tissue, by gripping scar tissue between thumbs and index fingers and moving } \\
\text { hands in opposite direction }\end{array}$ \\
\hline Exercise schemes & $\begin{array}{l}\text { To improve muscle flexibility, endurance, } \\
\text { and strength and active shoulder mobility } \\
\text { and to stimulate lymphatic transport }\end{array}$ & $\begin{array}{l}\text { Average } 10 \text { minutes per session. } 10 \text { exercise schemes built steadily and incrementally in difficulty. } \\
\text { Each exercise scheme consisted of exercise with proprioceptive neuromuscular facilitation ( }(3 \times) \text {; } \\
\text { active stretching of breast muscles }(3 \times 15 \mathrm{~s}) \text {; four different exercises-for example, scheme } 1 \text { : } \\
\text { 1) lying supine and both hands on shoulder, perform anteflexion of both shoulders; } 2 \text { ) same, but } \\
\text { abduction; 3) same, but circumduction; } 4 \text { ) lying supine, hands crossed, elbows straight and } 90^{\circ} \\
\text { anteflexion of shoulder, perform protraction of both shoulders. Scheme } 5: 1) \text { standing, holding } \\
\text { stick in both hands on buttock with straight elbows, perform retroflexion of both shoulders; } 2) \\
\text { same, but on thighs, perform anteflexion of shoulders; } 3 \text { ) same and } 90^{\circ} \text { anteflexion of shoulder, } \\
\text { perform horizontal abduction of affected shoulder; } 4 \text { ) standing, holding stick in both hands at } \\
\text { shoulder height, straighten elbows above head. At start of each exercise scheme, exercises } \\
\text { were performed } 7 \times \text {, then } 10 \times \text {, then } 10 \times \text { slow and } 5 \times \text { fast, and finally } 10 \times \text { slow and } 10 \times \text { fast. } \\
\text { Patients were asked to perform exercises from scheme twice/day at home, as taught during } \\
\text { treatment sessions }\end{array}$ \\
\hline
\end{tabular}


Table 2| Overview of primary and secondary outcome parameters and assessment methods in patients after unilateral axillary lymph node dissection for breast cancer

Outcome parameter

Measurement method

Primary

Cumulative incidence of arm lymphoedema, defined as $\geq 200 \mathrm{~mL}$ increase

Arm volume with water displacement method

Time to develop arm lymphoedema, defined as $\geq 200 \mathrm{~mL}$ increase Arm volume with water displacement method

\section{Secondary}

Cumulative incidence of arm lymphoedema, defined as $\geq 2 \mathrm{~cm}$ increase at two or more adjacent places Arm circumferences with measurement device

Time to develop arm lymphoedema, defined as $\geq 2 \mathrm{~cm}$ increase at two or more adjacent places Arm circumferences with measurement device

Point prevalence of arm lymphoedema, defined as $\geq 200 \mathrm{~mL}$ increase Arm volume with water displacement method

Point prevalence of arm lymphoedema, defined as $\geq 2 \mathrm{~cm}$ increase at two or more adjacent places Arm circumferences with measurement device

Point prevalence of subjective arm lymphoedema Author developed questionnaire

Increase of arm volume

Arm volume with water displacement method

Health related quality of life SF-36 
Table 3| Characteristics of patients according to treatments to prevent development of lymphoedema related to breast cancer. Figures are numbers (percentage) of patients unless specified otherwise

\begin{tabular}{|c|c|c|}
\hline & $\begin{array}{l}\text { Intervention (guidelines, exercise, manual } \\
\text { drainage; } n=77 \text { ) }\end{array}$ & Control (guidelines, exercise; $n=81$ ) \\
\hline Men & $1(1)$ & $1(1)$ \\
\hline Women & $78(99)$ & $80(99)$ \\
\hline Mean (SD) age (years) & $55.8(12.5)$ & $54.5(11.1)$ \\
\hline Mean (SD) BMI & $26.6(5.4)$ & $26.2(5.4)$ \\
\hline $\begin{array}{l}\text { Median (IQR) increase in arm volume before start of allocated } \\
\text { treatment }(\mathrm{mL})\end{array}$ & $8(-34-56)$ & $8(-16-64)$ \\
\hline $\begin{array}{l}\text { Mean (SD) interval between surgery and start of allocated treatment } \\
\text { (days) }\end{array}$ & $40(8)$ & $34(12)$ \\
\hline \multicolumn{3}{|l|}{ Mean (SD) No of sessions: } \\
\hline Exercise therapy & $28(6)$ & $28(8)$ \\
\hline Manual lymph drainage & $34(7)$ & 0 \\
\hline Mean (SD) No of lymph nodes & $19(6)$ & $18(6)$ \\
\hline \multicolumn{3}{|l|}{ Type of breast surgery: } \\
\hline Mastectomy & $52(66)$ & $56(69)$ \\
\hline Breast conserving & $27(34)$ & $25(31)$ \\
\hline Surgery on dominant side & $47(60)$ & $44(54)$ \\
\hline \multicolumn{3}{|l|}{ Level of axillary surgery: } \\
\hline 1 & $2(3)$ & $0(0)$ \\
\hline $\mathrm{I}-\mathrm{II}$ & $43(54)$ & $54(67)$ \\
\hline $\mathrm{I}-\mathrm{III}$ & $34(43)$ & $27(33)$ \\
\hline \multicolumn{3}{|l|}{ Tumour size : } \\
\hline рT0 & $1(1)$ & $0(0)$ \\
\hline pT1 & $21(27)$ & $26(32)$ \\
\hline pT2 & $38(48)$ & $39(48)$ \\
\hline pT3 & $13(17)$ & $12(15)$ \\
\hline pT4 & $6(8)$ & $4(5)$ \\
\hline \multicolumn{3}{|l|}{ Lymph node stage: } \\
\hline pNO & $23(29)$ & $25(31)$ \\
\hline $\mathrm{pN} 1$ & $36(46)$ & $39(48)$ \\
\hline $\mathrm{pN} 2$ & $11(14)$ & $9(11)$ \\
\hline $\mathrm{pN} 3$ & $9(11)$ & $8(10)$ \\
\hline Radiotherapy, IMC and medial supraclavicular & $69(87)$ & $67(83)$ \\
\hline Radiotherapy, axilla & $8(10)$ & $5(6)$ \\
\hline Chemotherapy & $50(63)$ & $58(72)$ \\
\hline Neo-adjuvant chemotherapy & $14(18)$ & $14(17)$ \\
\hline Trastuzumab & $14(18)$ & $7(9)$ \\
\hline Endocrine treatment & $55(70)$ & $66(82)$ \\
\hline
\end{tabular}

BMI=body mass index; IQR=interquartile range; IMC=internal mammary chain. 
Table 4| Comparison of cumulative incidence and point prevalence of arm lymphoedema after surgery for breast cancer at 3, 6, and 12 months for different definitions according to treatments to prevent lymphoedema

\begin{tabular}{|c|c|c|c|c|}
\hline Definition of lymphoedema & $\begin{array}{l}\text { Intervention (guidelines, exercise, manual drainage; } \\
\qquad \mathrm{n}=77 \text { ) }\end{array}$ & Control (guidelines, exercise; $\mathbf{n = 8 1}$ ) & Odds ratio $(95 \% \mathrm{Cl})$ & P value ${ }^{*}$ \\
\hline \multicolumn{5}{|l|}{ Primary outcome parameter } \\
\hline \multicolumn{5}{|c|}{ Cumulative incidence, $\geq 200 \mathrm{~mL}$ increase: } \\
\hline At 3 months & $8(10 \%)$ & $6(7 \%)$ & $1.4(0.5$ to 4.4$)$ & 0.51 \\
\hline At 6 months & $11(14 \%)$ & $12(15 \%)$ & $0.9(0.4$ to 2.3$)$ & 0.93 \\
\hline At 12 months $\dagger$ & $18(24 \%)$ & $15(19 \%)$ & $1.3(0.6$ to 2.9$)$ & 0.45 \\
\hline \multicolumn{5}{|c|}{ Secondary outcome parameters } \\
\hline \multicolumn{5}{|c|}{ Cumulative incidence, $\geq 2 \mathrm{~cm}$ increase: } \\
\hline At 3 months & $8(10 \%)$ & $6(7 \%)$ & $1.4(0.5$ to 4.4$)$ & 0.51 \\
\hline At 6 months & $12(16 \%)$ & $11(14 \%)$ & $1.2(0.5$ to 2.8$)$ & 0.72 \\
\hline At 12 months $\dagger$ & $20(27 \%)$ & $16(20 \%)$ & $1.4(0.7$ to 3.0$)$ & 0.35 \\
\hline \multicolumn{5}{|c|}{ Point prevalence, $\geq 200 \mathrm{~mL}$ increase: } \\
\hline At 3 months & $5(7 \%)$ & $3(4 \%)$ & $1.8(0.4$ to 7.8$)$ & 0.43 \\
\hline At 6 months & $4(5 \%)$ & $8(10 \%)$ & $0.5(0.1$ to 1.7$)$ & 0.28 \\
\hline At 12 months $\dagger$ & $9(12 \%)$ & $8(10 \%)$ & $1.2(0.4$ to 3.3$)$ & 0.71 \\
\hline \multicolumn{5}{|c|}{ Point prevalence, $\geq 2 \mathrm{~cm}$ increase: } \\
\hline At 3 months & $5(7 \%)$ & $4(5 \%)$ & $1.3(0.3$ to 5.2$)$ & 0.67 \\
\hline At 6 months & $6(8 \%)$ & $8(10 \%)$ & $0.8(0.3$ to 2.3$)$ & 0.65 \\
\hline At 12 months $\dagger$ & $11(15 \%)$ & $7(9 \%)$ & $1.8(0.6$ to 4.8$)$ & 0.27 \\
\hline \multicolumn{5}{|l|}{ Point prevalence, subjective: } \\
\hline At 3 months & $14(18 \%)$ & $9(11 \%)$ & $1.8(0.7$ to 4.4$)$ & 0.21 \\
\hline At 6 months & $12(16 \%)$ & $8(10 \%)$ & $1.7(0.6$ to 4.4$)$ & 0.28 \\
\hline At 12 months $\dagger$ & $18(24 \%)$ & $14(18 \%)$ & 1.5 (0.7 to 3.2$)$ & 0.34 \\
\hline
\end{tabular}

${ }^{*}$ For logistic regression analysis.

†75 in intervention group, 79 in control group. 
Table 5| Comparison of changes in difference in arm volume between affected and healthy arm compared with difference in volume before surgery for breast cancer and comparison of mental and physical health related quality of life (secondary outcomes) between groups at each follow-up according to treatments to prevent lymphoedema

Time after surgery Intervention (guidelines, exercise, manual drainage; $n=77$ ) Control (guidelines, exercise; $n=81$ ) $P$ value ${ }^{\star}$ Change in difference in arm volume $(\mathrm{mL})$ :

\begin{tabular}{|c|c|c|c|}
\hline At 3 months & $29(82)$ & $18(101)$ & 0.69 \\
\hline At 6 months & $58(104)$ & $31(114)$ & 0.42 \\
\hline At 12 months $\dagger$ & $34(158)$ & $45(111)$ & 0.97 \\
\hline \multicolumn{4}{|l|}{ Quality of life: } \\
\hline \multicolumn{4}{|c|}{ Mental health (maximum score 100): } \\
\hline At 3 months & $72(34)$ & $69(38)$ & 0.70 \\
\hline At 6 months & $74(42)$ & $68(33)$ & 0.59 \\
\hline At 12 months $†$ & $79(36)$ & $81(32)$ & 0.74 \\
\hline \multicolumn{4}{|c|}{ Physical health (maximum score 100): } \\
\hline At 3 months & $56(27)$ & $56(38)$ & 0.54 \\
\hline At 6 months & $63(40)$ & $58(36)$ & 0.81 \\
\hline At 12 months $†$ & $74(37)$ & 77 (35) & 0.48 \\
\hline
\end{tabular}

${ }^{*}$ For Mann-Whitney U test.

†75 in intervention group, 79 in control group. 


\section{Figures}

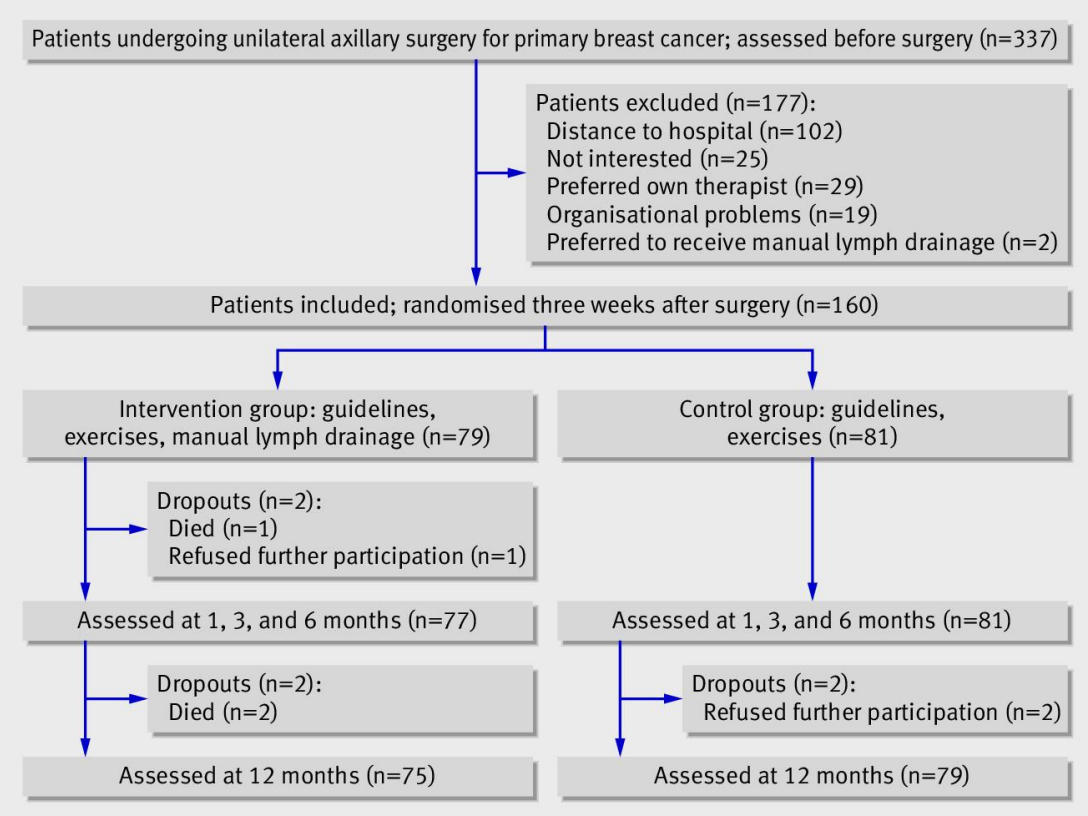

Fig 1 Trial profile of patients with breast cancer undergoing axillary lymph node dissection according to allocation to intervention (guidelines, exercise therapy, and manual lymph drainage) or control (guidelines, exercise therapy)

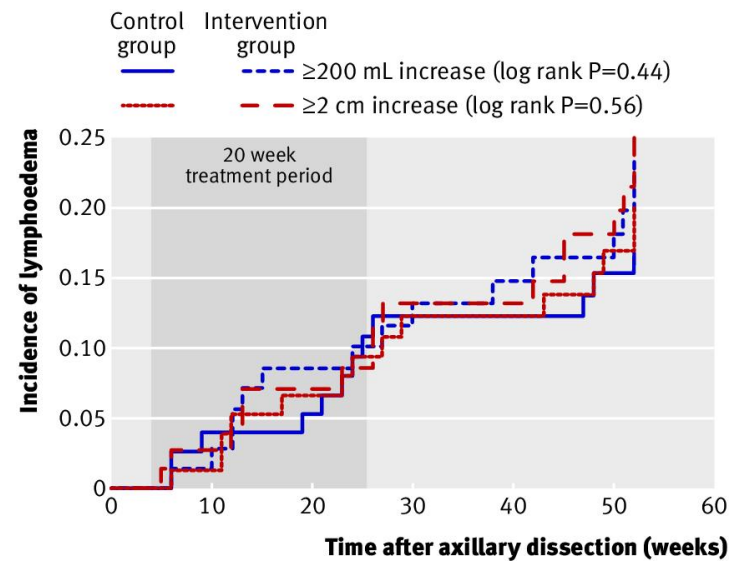

Fig 2 Time to develop lymphoedema after axillary lymph node dissection for breast cancer in 160 patients for two definitions of lymphoedema: increase $\geq 200 \mathrm{~mL}$ in volume or increase $\geq 2 \mathrm{~cm}$ in circumference at two adjacent points

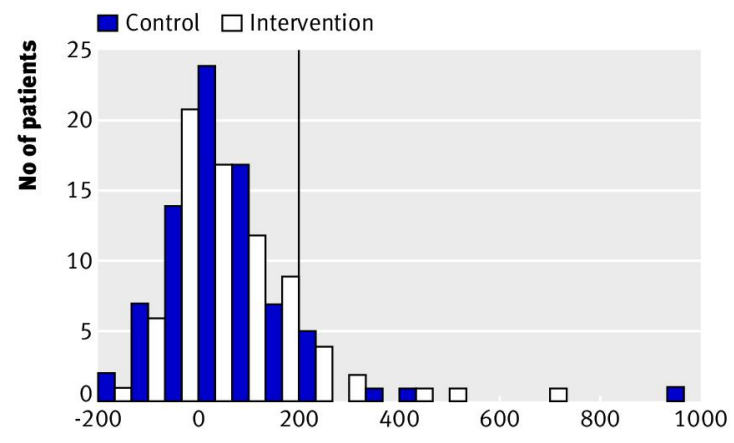

Change in arm volume $(\mathrm{mL})$ 
Fig 3 Change in difference in arm volume between affected and healthy side compared with preoperative difference 12 months after surgery for breast cancer. Vertical line corresponds to cut-off value for diagnosis of lymphoedema ( $\geq 200 \mathrm{~mL}$ increase)

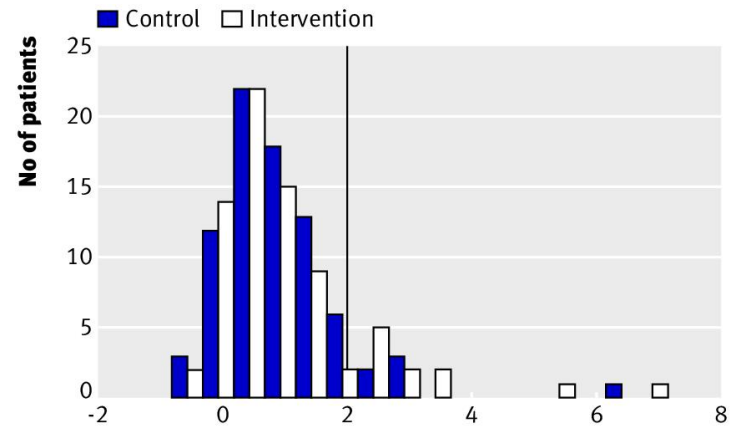

Maximum increase in arm circumference
at two adjacent measurement points (cm)

Fig 4 Maximum increase in difference in arm circumference between affected and healthy side at two or more adjacent measurement points compared with preoperative difference 12 months after surgery for breast cancer. Vertical line corresponds to cut-off value for diagnosis of lymphoedema ( $\geq 2 \mathrm{~cm}$ increase at two or more adjacent measurement points) 